kidney biology through negative regulation of WNT/ $\beta$-catenin signaling, then we should be able to ascribe a WNT-related function to the $\mathrm{C}$ terminus of WTX. Therefore, we mapped the domain of WTX that interacts with $\beta$-catenin and found that $\beta$-catenin purified with full-length WTX and the C-terminal half of WTX (WTX-C), but interacted poorly with the N-terminal half (WTX-N) (Fig. 2D and fig. S2 and supporting online text). As additional confirmation, we used our TAP-LC-MS/MS analysis on cells expressing pGlue-WTX-C and found both $\beta$-TrCP and $\beta$-catenin within the protein complex (table S1). Thus, mutational alteration of WTX in Wilms tumor likely reduces its interaction with $\beta$-catenin and $\beta$-TrCP.

The direct binding of WTX to both $\beta$-catenin and to its E3 ubiquitin ligase adaptor, $\beta-\operatorname{TrCP}$, suggests that WTX regulates $\beta$-catenin degradation. We tested this hypothesis using cell-free Xenopus egg extracts, an experimental system that allows quantitative monitoring of $\beta$-catenin ubiquitination and degradation (11). The addition of recombinant glutathione $S$-transferase (GST) in complex with WTX protein increased the rate of $\beta$-catenin ubiquitination, but GST control did not (Fig. 3, A and B, and fig. S3). Inhibition of GSK3 by lithium chloride $(\mathrm{LiCl})$ suppressed $\beta$-catenin ubiquitination in the presence of GST and GST-WTX. As a scaffold protein, AXIN nucleates the GSK3-CK1-APC phosphorylation complex and thereby dramatically increases $\beta$-catenin turnover in Xenopus extracts (11). When WTX and AXIN1 were added to the extracts individually, each increased the rate of $\beta$-catenin degradation (Fig. 3C). When WTX and AXIN1 were added together, the rate of $\beta$-catenin degradation was more rapid than observed with either alone. These data suggest that WTX negatively regulates WNT signaling by promoting $\beta$-catenin ubiquitination.

If WTX promotes $\beta$-catenin degradation, then suppressing WTX expression should activate $\mathrm{WNT} / \beta$-catenin signaling in mammalian cells. To test this prediction, we measured the activity of a $\beta$-catenin-dependent transcriptional reporter after small interfering RNA (siRNA)-mediated silencing of WTX. Specifically, HEK293T human embryonic kidney cells and RKO human colon carcinoma cells were transduced with lentiviruses encoding a firefly luciferase-based $\beta$-catenin-activated reporter (pBAR), along with Renilla luciferase (Renilla-Luc) under the control of the constitutively active thymidine kinase promoter for normalization. To validate the dynamic range of this reporter system, stably transduced cell lines were treated with WNT3aconditioned medium, which activated the reporter by a factor of 100 to 300 (Fig. 3, D and E). As a control, we showed that siRNAs directed against $\beta$-catenin abolished this WNT3a-induced reporter activity in both cell lines (fig. S4 and supporting online text). Using this assay system, we found that two different siRNAs targeting WTX produced an increase in WNT3a-induced reporter activity in both cell types. Furthermore, in RKO-pBAR/Renilla cells, siRNA-mediated silencing of WTX, AXIN1 and 2, or $\beta$-TrCP1 and 2 synergized with a GSK3 inhibitor, $\left(2^{\prime} Z, 3^{\prime} E\right)-6$ bromoindirubin- 3 '-oxime, to activate the $\mathrm{pBAR}$ reporter (fig. S4). These data suggest that WTX is a negative regulator of $\mathrm{WNT} / \beta$-catenin signal transduction in mammalian cells.

We next tested whether silencing of WTX with siRNAs increases $\beta$-catenin levels in cells. In RKO cells, $\beta$-catenin does not localize to the plasma membrane, whereas in other cell types, such as HEK293T cells, it resides with a relatively long half-life at the inner surface of the plasma membrane. Thus, in the absence of membraneassociated $\beta$-catenin, total cellular levels of $\beta$-catenin in RKO cells are very low, which allows study of cytoplasmic and nuclear $\beta$-catenin stability in response to experimental perturbation. We transiently transfected RKO cells with siRNAs targeting WTX, $\beta$-catenin, AXIN1 and 2, or $\beta$-TrCP1 and 2. Silencing of WTX, AXIN1 and 2, or $\beta-\operatorname{TrCP} 1$ and 2 , but not $\beta$-catenin, was found to increase $\beta$-catenin levels, as determined by immunoblot analysis (Fig. 3F). Thus, WTX is required in these cells as a negative regulator of both $\beta$-catenin protein stability and $\beta$-catenin-mediated transcription.

To extend these experiments to organisms, we performed gain-of-function experiments in Xenopus embryos and loss-of-function experiments in zebrafish (supporting online text). Ectopic activation of $\mathrm{WNT} / \beta$-catenin signaling by injection of Xenopus Wnt8 mRNA in Xenopus embryo ventral blastomeres induced duplication of the embryonic axis, yielding two-headed tadpoles (fig. S5). Injection of WTX mRNA blocked Xenopus Wnt8-induced axis duplication. In developing zebrafish embryos, ectopic activation of WNT/ $\beta$-catenin signaling leads to anterior truncations. When we silenced endoge- nous zebrafish wtx expression, we observed anterior truncations and the activation of a $\mathrm{WNT} / \beta$-catenin reporter gene (fig. S5). These results suggest that WTX is a negative regulator of $\mathrm{WNT} / \beta$-catenin signaling in vivo.

In summary, these data establish that the cancer-associated WTX protein is a required component of the $\beta$-catenin destruction complex. Furthermore, our data underscore the power of proteomic approaches for identifying new components of cellular signal transduction pathways that may ultimately provide important mechanistic insights into human disease.

\section{References and Notes}

1. D. Kimelman, W. Xu, Oncogene 25, 7482 (2006).

2. C. Y. Logan, R. Nusse, Annu. Rev. Cell Dev. Biol. 20, 781 (2004).

3. K. Willert, K. A. Jones, Genes Dev. 20, 1394 (2006).

4. R. T. Moon, A. D. Kohn, G. V. De Ferrari, A. Kaykas Nat. Rev. Genet. 5, 691 (2004).

5. H. Clevers, Cell 127, 469 (2006)

6. Materials and methods are available as supporting material on Science Online.

7. S. Angers et al., Nat. Cell Biol. 8, 348 (2006).

8. M. N. Rivera et al., Science 315, 642 (2007).

9. R. Koesters et al., Cancer Res. 59, 3880 (1999).

10. R. Koesters, F. Niggli, M. von Knebel Doeberitz, T. Stallmach, J. Pathol. 199, 68 (2003).

11. A. Salic, E. Lee, L. Mayer, M. W. Kirschner, Mol. Cell 5, 523 (2000).

12. Purified $\beta$-catenin was a kind gift from W. Xu, University of Washington, Seattle. C.H. is supported by a postdoctoral F32 NIH National Research Service Award training grant.

\section{Supporting Online Material}

www.sciencemag.org/cgi/content/full/316/5827/1043/DC1 Materials and Methods

SOM Text

Figs. $\mathrm{S} 1$ to $\mathrm{S} 6$

Tables S1 and S2

References

20 February 2007; accepted 30 March 2007 $10.1126 /$ science/1141515

\title{
Revisiting the Role of the Mother Centriole in Centriole Biogenesis
}

\author{
A. Rodrigues-Martins, ${ }^{1,2}$ M. Riparbelli, ${ }^{3}$ G. Callaini, ${ }^{3}$ D. M. Glover, ${ }^{2 *}$ M. Bettencourt-Dias ${ }^{1,2 *}$
}

Centrioles duplicate once in each cell division cycle through so-called templated or canonical duplication. SAK, also called PLK4 (SAK/PLK4), a kinase implicated in tumor development, is an upstream regulator of canonical biogenesis necessary for centriole formation. We found that overexpression of SAK/PLK4 could induce amplification of centrioles in Drosophila embryos and their de novo formation in unfertilized eggs. Both processes required the activity of DSAS-6 and DSAS-4, two molecules required for canonical duplication. Thus, centriole biogenesis is a templatefree self-assembly process triggered and regulated by molecules that ordinarily associate with the existing centriole. The mother centriole is not a bona fide template but a platform for a set of regulatory molecules that catalyzes and regulates daughter centriole assembly.

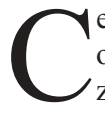
entrioles are essential for the formation of cilia and flagella and for the organization of the centrosome (1). Normally, centrioles duplicate in coordination with the cell cycle. A new centriole, the daughter, arises orthog- onally to each old one, the mother (1), in S phase. This led to the idea that the mother centriole templates the formation of the daughter $(2,3)$. However, daughter centrioles do not incorporate a substantial proportion of the mother (4), 
and centrioles can also form de novo when existing centrioles are naturally lost during development or are physically removed (5-7), questioning the idea of the mother centriole as a template.

SAK, also called PLK4 (SAK/PLK4), a kinase implicated in tumor development $(8)$, is an upstream regulator of canonical centriole duplication and is necessary for centriole formation (9, 10). The Caenorhabditis elegans ZYG1 kinase, a homolog of SAK, is part of a conserved module of proteins, which also includes SAS-6 and SAS-4, necessary for the normal centriole duplication cycle (11-16). ZYG-1 is an upstream regulator in that process $(17,18)$, a role consistent with formation of multiple centrioles in cultured cells following overexpression of active SAK kinase $(9,10)$. The generation of multiple centrioles associated with high SAK expression also occurs physiologically in the olfactory mucosa (19). The Drosophila egg contains all the proteins necessary to make $2^{13}$ centriole pairs (centrosomes) (20). Centrioles are naturally eliminated from the oocyte cytoplasm in the course of development and provided to the egg in the form of the basal body of the sperm (20-23). Thus, we studied the consequences of overexpressing SAK in a cytoplasm that either contained centrioles (the embryo) or lacked them (the unfertilized egg).

Embryos overexpressing SAK did not develop (24) (fig. S1A and Fig. 1A) and were filled with free asters of microtubules not associated with spindles (Fig. 1, B and D, and fig. S1B). Those asters were focused around Drosophila pericentrin-like protein (D-PLP)-containing structures, a centriolar and pericentriolar material (PCM) marker (25) (fig. S1B). These centrosomes first appeared in 15- to 30-min-old embryos (Fig. 1B) and spread to fill the entire embryo after 2 to 3 hours (Fig. 1, C and D). The observed supernumerary centrosomes led to abnormal mitotic progression and impaired embryonic development, as observed previously upon microtubule depolymerization by colchicine treatment (26). To address the origins of those centrosomes, we examined the very early stages of embryonic development in embryos overexpressing SAK. Both the sperm aster around the incoming basal body and the first mitotic spindle were normal (Fig. 1E). However, at anaphase or telophase of the first mitosis, we observed more than two centrosomes at each pole (Fig. 1E), an indication of the onset of centrosome amplification. No other centrosomes were seen in the embryo at this stage. Moreover,

${ }^{1}$ Instituto Gulbenkian de Ciência, Cell Cycle Regulation Laboratory, Rua da Quinta Grande, 6, P-2780-156 Oeiras, Portugal. ${ }^{2}$ Cancer Research UK, Cell Cycle Genetics Research Group, Department of Genetics, University of Cambridge, Downing Street, Cambridge CB2 3EH, UK. ${ }^{3}$ Department of Evolutionary Biology, University of Siena, Via A. Moro 4, I-53100 Siena, Italy.

*To whom correspondence should be addressed. E-mail: mdias@igc.gulbenkian.pt (M.B.-D.); dmg25@hermes.cam. ac.uk (D.M.G.) we estimated that a minimum of 3700 centrosomes (equivalent to 12 duplication cycles) were present after $60 \mathrm{~min}$ in embryos overexpressing SAK. After $60 \mathrm{~min}$, a wild-type embryo only showed 128 centrosomes. We observed duplicating centrioles in groups, suggesting they originated by duplication of a progenitor (Fig. 1, D and E, and fig. S1B). Thus, upon fertilization of

A
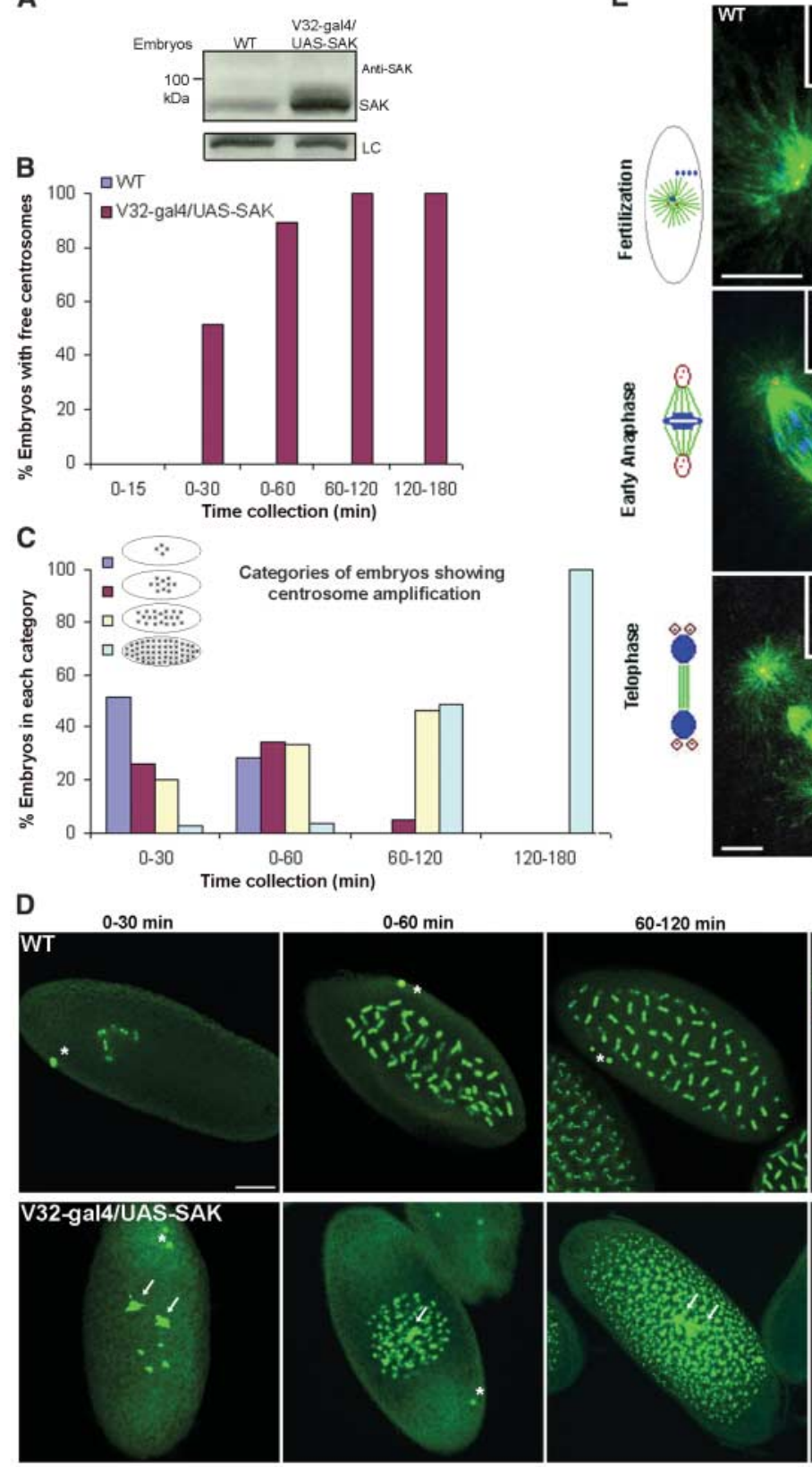

eggs overexpressing SAK, the basal body of the sperm enters an environment that promotes accelerated canonical duplication, overriding any existing controls that would normally couple the centrosome and chromosome cycles.

Uncoupling between centrosome and chromosome cycles occurs when embryos are arrested in S-phase-like conditions $(27,28)$. However,

\section{E}
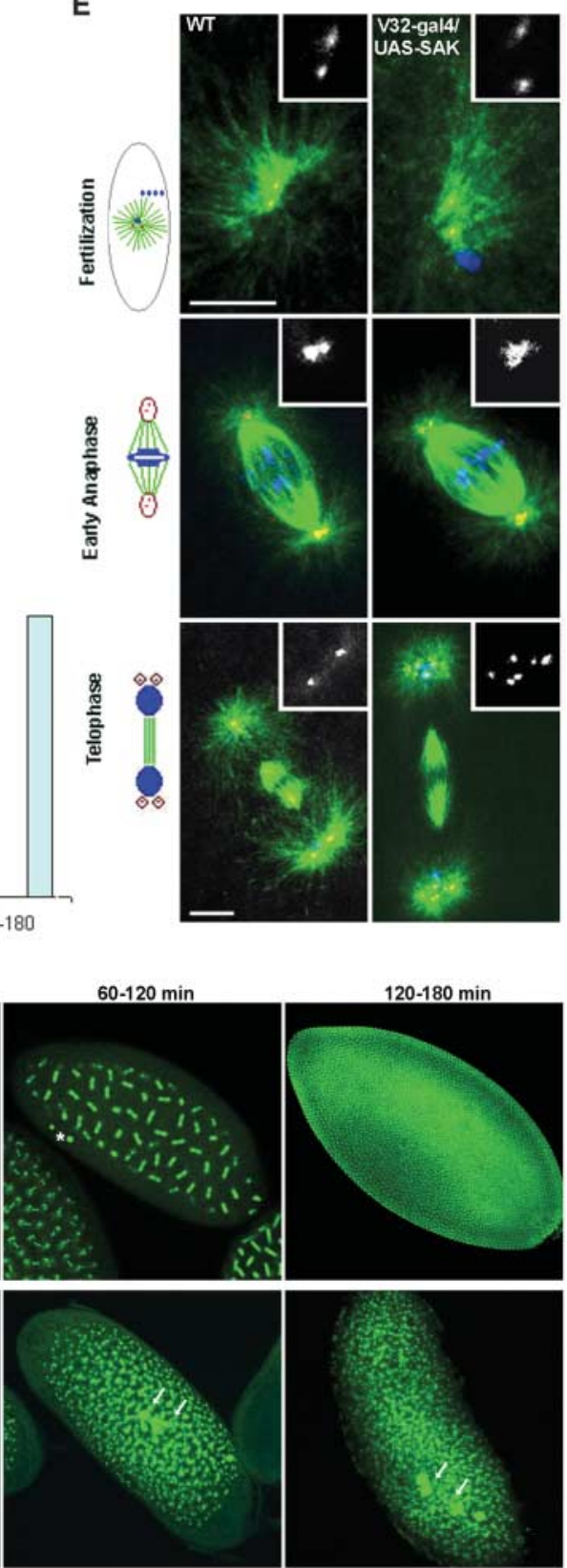

Fig. 1. Overexpression of SAK in Drosophila embryos leads to massive centrosome amplification. (A) Overexpression of upstream activation sequence (UAS)-SAK in embryos using the maternal driver V32gal4. LC indicates loading control; WT, wild type. (B) Embryos overexpressing SAK become progressively full of free centrosomes nucleating asters. (C and D) The spreading of the centrosomes follows the wildtype spindle axial expansion pattern. Categories were as follows [according to the area occupied by the centrosomes within the embryo (24)]: 0 to $2 \%, 2$ to $20 \%, 20$ to $60 \%$, and more than $60 \%$ area occupancy. $\alpha$-tubulin is shown in green. An average of 60 embryos were counted in each category. Asterisks indicate polar bodies. Arrows indicate spindles. Scale bar indicates $50 \mu \mathrm{m}$. (E) Centrosome amplification in embryos is observed at the end of first mitosis. $\gamma$-tubulin is shown in red; $\alpha$-tubulin, green; and DNA, blue. Scale bar, $10 \mu \mathrm{m}$. (Insets) $\gamma$-tubulin at $2 \times$. 
Fig. 2. SAK induces de novo centrosome formation in Drosophila eggs. (A) Overexpression of UAS-SAK in eggs using the maternal driver V32gal4. LC, loading control. (B) De novo centrosome formation starts after 30 min. (C and D) De novo centrosomes appear randomly in space [arrows in (D)]. Categories were as follows: 0 to $2 \%, 2$ to $60 \%$, and more than $60 \%$ area occupancy. An average of 67 eggs was counted in each category. Scale bar, $50 \mu \mathrm{m}$. (E) Meiosis II occurs normally in V32-gal4/UAS SAK eggs with no visible centrosomes $(n=110)$. $\mathrm{i}$ and ii indicate magnified fields. Scale bars, $50 \mu \mathrm{m}$ (left) and $10 \mu \mathrm{m}$ (inset) in each wild-type and overexpressing set. $\gamma$-tubulin, red; $\alpha$-tubulin green; and DNA, blue.

Fig. 3. De novo- and canonical-formed centrosomes show centriolar and centrosomal markers and are structurally normal. (A and B) Centrosomes in both 0 to 1 hour embryos and eggs overexpressing SAK contain $\gamma$-tubulin, DSAS-6, DSAS4, and SAK. (A) $\gamma$-tubulin, red; $\alpha$-tubulin, green in left and red in middle and right; green fluorescent protein (GFP)-DSAS6, green; DSAS4-GFP, green; and DNA, blue. Scale bar, $10 \mu \mathrm{m}$. (B) SAK, green; D-PLP, red; and $\alpha$-tubulin, blue. Scale bar, $10 \mu \mathrm{m}$. (C) De novo- and canonicalformed centrosomes are structurally normal by transmission electron microscopy. Bottom images are higher-magnification examples of centrioles in each condition. Asterisks indicate duplicating centrioles. Scale bars as indicated.

A

B

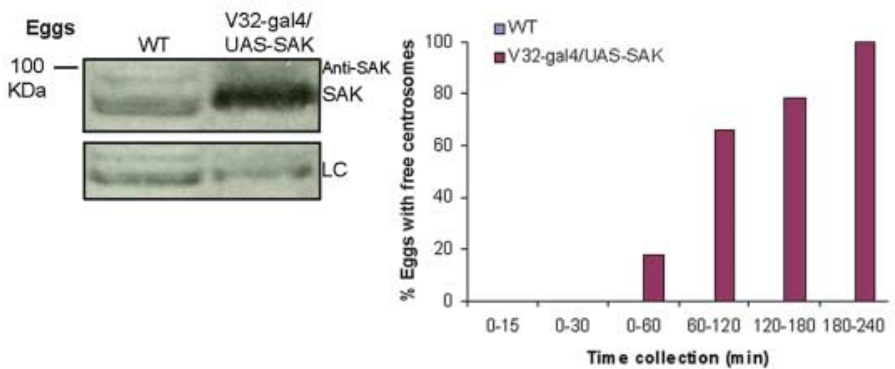

D
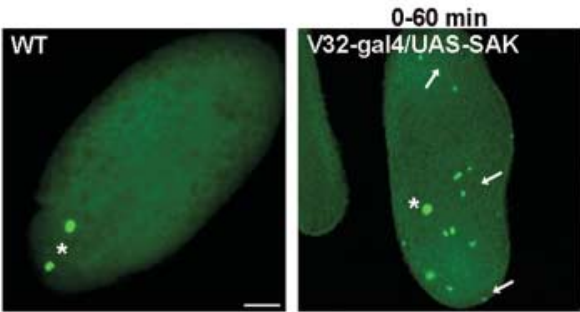

60-120 min
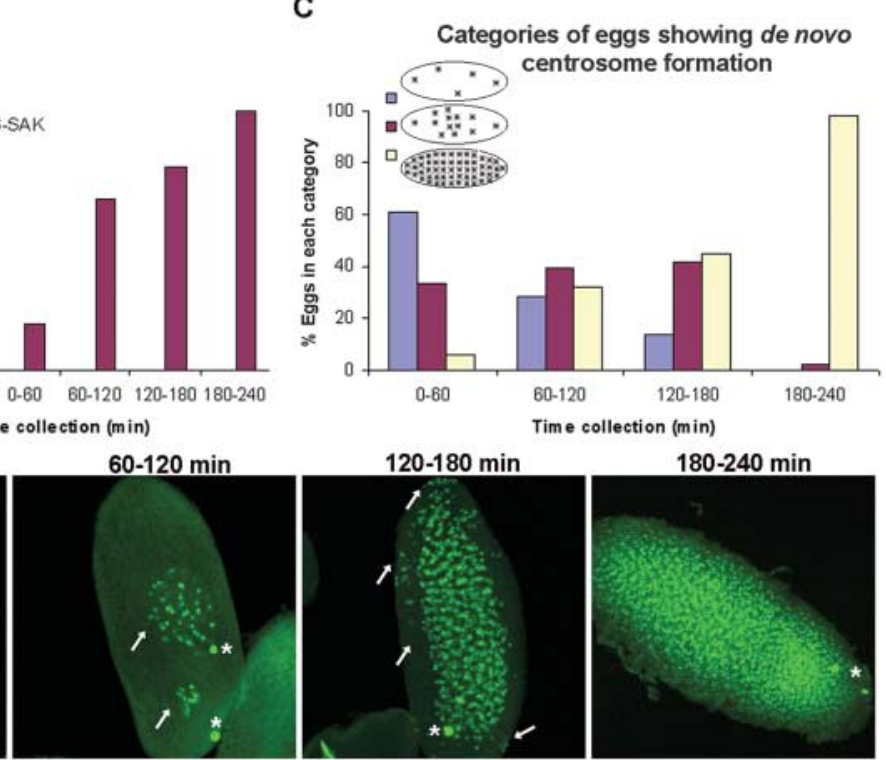

E
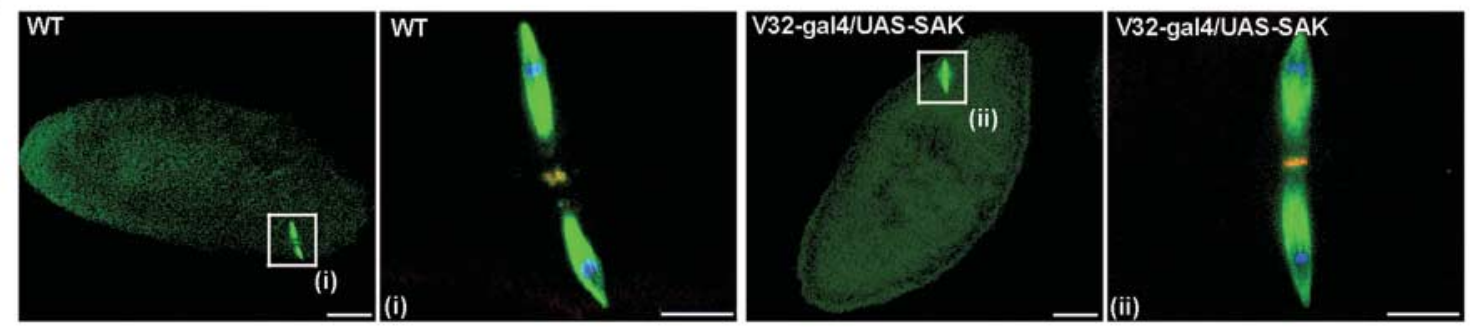

A
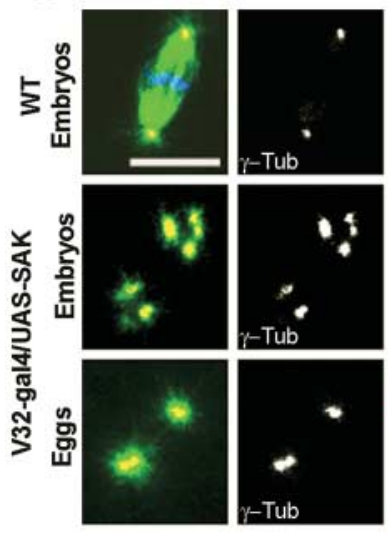
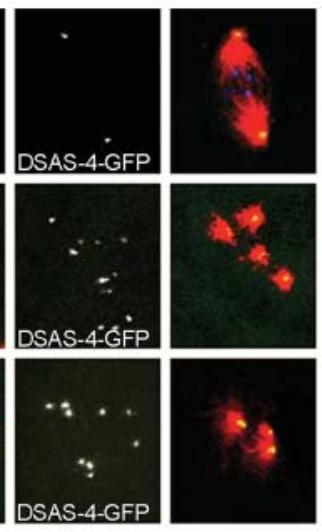

C

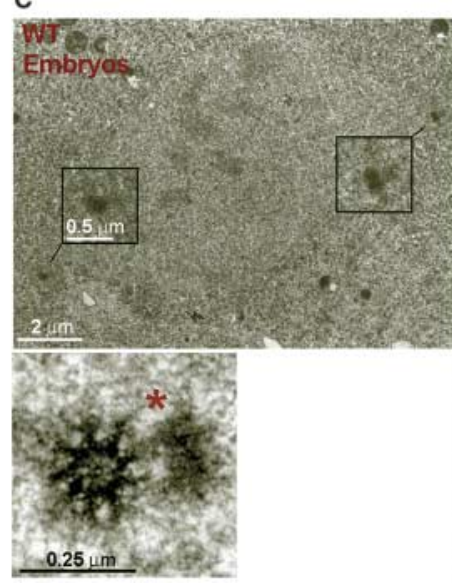

B

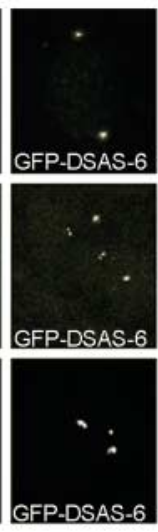

$180-240 \mathrm{~min}$

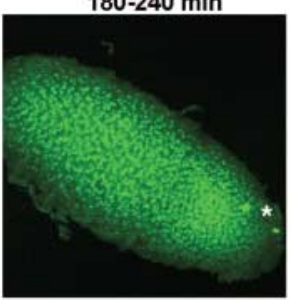

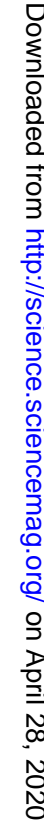


this did not seem to be so in this case, because proliferating cell nuclear antigen (PCNA) (29), which appears early in $\mathrm{S}$ phase, was not detected in DNA of SAK-overexpressing embryos (fig. S2).

We next asked whether SAK could promote centriolar assembly in the absence of centrioles. Centrioles were lost normally in oocytes overexpressing SAK (fig. S3). Yet observations of unfertilized eggs at varying developmental intervals revealed free centrosomes in eggs overexpressing SAK that had exited meiosis II (Fig. 2 and fig. S4) but never in wild-type eggs. Thus, in the absence of a basal body provided by the sperm, SAK can induce de novo formation of centrosomes. Whereas in embryos centrosomes appeared in a single cluster in the first mitotic spindle and spread throughout the cytoplasm (Fig. 1, D and E), in unfertilized eggs they appeared scattered at random positions, including at the anterior and posterior poles (Fig. 2D, arrows). The formation of the first centrioles started later in eggs than in embryos [at $30 \mathrm{~min}, 0$ amplification in eggs versus $51 \%$ amplification in embryos; after 1 hour, the

A

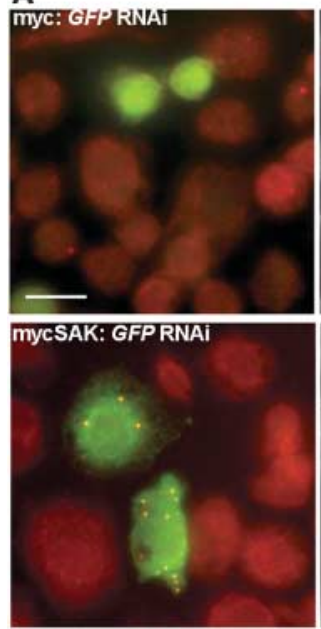

myCSAK: DSAS-6 RNAi
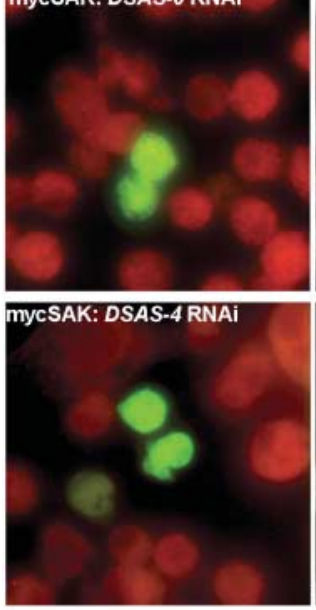

B
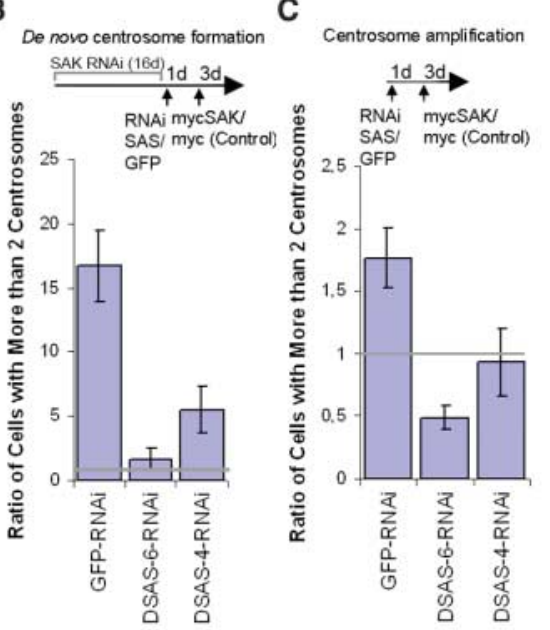

Fig. 4. Canonical and de novo SAK-induced centrosome amplification is dependent on DSAS-6 and DSAS-4. (A and B) Overexpression of myc-SAK in S2 cells devoid of centrosomes leads to de novo centrosome formation that is dependent on DSAS-6 and DSAS-4. Cells were submitted to SAK RNAi for 16 days (four retransfections), leading to a population of cells where $80 \%$ showed no centrioles. Cells were then transfected with DNA (myc or mycSAK) and double-stranded RNA (GFP, DSAS4, or DSAS6) according to the scheme in (B) and stained for myc and D-PLP. (A) Myc and myc-SAK, green, and D-PLP, red. (B) The number of centrosomes per myc-positive cell was counted. The proportion of cells showing more than two centrosomes in each condition is shown as a ratio over the control (cells transfected with myc). (C) Overexpression of mycSAK in S2 cells leads to centrosome amplification that is dependent on DSAS-6 and DSAS-4. The same experiment as in (B) was performed, but this time in a population of cells not submitted previously to SAK RNAi. Scale bar, $10 \mu \mathrm{m}$. A minimum of 100 cells were counted for each condition in each of three independent experiments. Error bars indicate standard deviation. (fig. S9 and Fig. 3C), a result suggesting that SAK-induced centrioles can duplicate.

Our results show that SAK is sufficient to induce both canonical and de novo centriole biogenesis. If both rely on self-assembly of the structure, we would predict the use of the same regulatory molecules. We examined the dependency of SAK-promoted centriole biogenesis on DSAS-4 and DSAS- 6 . We took advantage of the fact that centrioles can be eliminated from Drosophila tissue culture cells (10). After depletion of SAK in four rounds of RNA interference (RNAi) over a period of 16 days, more than $80 \%$ of the cells lacked centrioles, presumably because the remainder are diluted in each division cycle (Fig. 4A) (10). Subsequent overexpression of SAK led to a clear increase in the number of cells with several centrosomes (from 4 to $48 \%$ ) (Fig. 4, A and B). Depletion of DSAS-6 or DSAS-4 prevented SAKinduced centrosome biogenesis in cells with and without centrioles (Fig. 4, A to C, and fig. S10).

Our results suggest that centriole biogenesis is a template-free self-assembly process that is locally triggered and regulated by molecules such as SAK, DSAS-6, and DSAS-4. What could be the role of the mother centriole? The presence of SAK at the centriole (Fig. 3B and fig. S7) $(9,10)$ and the fact that assembly is faster in the presence of centrioles (fig. S5) $(5,6)$ suggest that the mother centriole is not a bona fide template but a platform for regulatory molecules, hence catalyzing and regulating daughter centriole assembly. The establishment of that platform is probably less efficient in the absence of centrioles. The mother centriole could in principle establish a temporally and spatially regulated gradient of SAK activity, as demonstrated for RanGTP, a small guanosine triphosphatase involved in spindle assembly (32), perhaps counteracted in the cytoplasm by other molecules. Our data and that of other groups also point to a role for centrioles in regulating total centriole number, because their presence precludes de novo formation (Fig. 1D and fig. S11) $(5,6)$. This is true even in a large embryo $(\sim 800 \mu \mathrm{m})$ containing very large amounts of SAK (Fig. 1D). Whether this indicates sequestering of active SAK or its substrates in existing centriolar structures or an active inhibitory effect of centrioles upon de novo assembly requires further study.

The regulation of SAK activity is essential in the control of centriole number (fig. S11) and may be a parameter that is regulated according to cellular needs, because multiciliated cells of the respiratory tract have high SAK levels (19). The activity of SAK may be inhibited in the acentriolar female meiosis, as de novo centrosome formation only occurs after meiosis exit in eggs overexpressing SAK (Fig. 2). Drosophila eggs and embryos should provide an ideal experimental system for further analyses of the control of centriole biogenesis and how it may go awry in cancer.

\section{References and Notes}

1. M. Delattre, P. Gonczy, J. Cell Sci. 117, 1619 (2004).

2. W. F. Marshall, J. Cell. Biochem. 100, 916 (2007). 
3. J. Gall, in Centrosomes in Development and Disease, E. A. Nigg, Ed. (Wiley-VCH, Weinheim, Germany, 2004)

4. R. S. Kochanski, G. G. Borisy, J. Cell Biol. 110, 1599 (1990).

5. S. La Terra et al., J. Cell Biol. 168, 713 (2005).

6. W. F. Marshall, Y. Vucica, J. L. Rosenbaum, Curr. Biol. 11, 308 (2001).

7. M. G. Riparbelli, G. Callaini, Dev. Biol. 260, 298 (2003).

8. M. A. Ko et al., Nat. Genet. 37, 883 (2005)

9. R. Habedanck, Y. D. Stierhof, C. J. Wilkinson, E. A. Nigg, Nat. Cell Biol. 7, 1140 (2005)

10. M. Bettencourt-Dias et al., Curr. Biol. 15, 2199 (2005).

11. A. Dammermann et al., Dev. Cell 7, 815 (2004)

12. S. Leidel, M. Delattre, L. Cerutti, K. Baumer, P. Gonczy, Nat. Cell Biol. 7, 115 (2005).

13. K. F. O'Connell et al., Cell 105, 547 (2001).

14. S. Leidel, P. Gonczy, Dev. Cell 4, 431 (2003).

15. M. Kirkham, T. Muller-Reichert, K. Oegema, S. Grill, A. A. Hyman, Cell 112, 575 (2003).

16. R. Basto et al., Cell 125, 1375 (2006).

17. M. Delattre, C. Canard, P. Gonczy, Curr. Biol. 16, 1844 (2006).

18. L. Pelletier, E. O'Toole, A. Schwager, A. A. Hyman, T. Muller-Reichert, Nature 444, 619 (2006).
19. C. Fode, B. Motro, S. Yousefi, M. Heffernan, J. W. Dennis, Proc. Natl. Acad. Sci. U.S.A. 91, 6388 (1994).

20. V. Foe, G. M. Odell, B. Edgar, in The Development of Drosophila melanogaster, M. Bate, A. Martinez Arias, Eds. (Cold Spring Harbor Laboratory Press, Cold Spring Harbor, NY, 1993), pp. 149-287.

21. C. Gonzalez, G. Tavosanis, C. Mollinari, J. Cell Sci. 111 2697 (1998).

22. A. P. Mahowald, ]. M. Strassheim, J. Cell Biol. 45, 306 (1970).

23. J. Januschke et al., Development 133, 129 (2006).

24. Materials and methods are available as supporting online material on Science Online.

25. M. Martinez-Campos, R. Basto, J. Baker, M. Kernan, J. W. Raff, ]. Cell Biol. 165, 673 (2004).

26. D. Perez-Mongiovi, N. Malmanche, H. Bousbaa, C. Sunkel, Development 132, 4509 (2005)

27. M. Freeman, D. M. Glover, Genes Dev. 1, 924 (1987).

28. F. L. Shamanski, T. L. Orr-Weaver, Cell 66, 1289 (1991).

29. M. Yamaguchi, T. Date, A. Matsukage, J. Cell Sci. 100, 729 (1991).

30. I. Belecz, C. Gonzalez, ]. Puro, J. Szabad, Curr. Biol. 11, 136 (2001).

31. G. Goshima et al., Science 316, 417 (2007); published online 5 April 2007 (10.1126/science.1141314).
32. P. Bastiaens, M. Caudron, P. Niethammer, E. Karsenti, Trends Cell Biol. 16, 125 (2006).

33. We thank C. Ferreira and I. Ferreira for help with experiments; D. Johnston, R. Basto, and J. Raff for reagents; R. Martinho, J. Leal, M. Gatt, R. Kuriyama, and anonymous reviewers for comments on the manuscript; the Drosophila Instituto Gulbenkian de Ciência (IGC) community, G. Goshima, and the M.B.-D. and D.M.G. groups for discussions; thebestgene.com for making transgenic flies; and the IGC imaging unit for help with image acquisition. We are grateful for grants from Cancer Research UK, Fundação Calouste Gulbenkian, and Fundação para a Ciência e Tecnologia (POCI2010) and for an International Joint Project grant from the Royal Society for collaboration between the M.B.-D. and D.M.G. groups.

Supporting Online Material

www.sciencemag.org/cgi/content/full/1142950/DC1

Materials and Methods

Figs. S1 to S11

References

23 March 2007; accepted 17 April 2007

Published online 26 April 2007;

10.1126/science.1142950

Include this information when citing this paper.

\section{Combined Action of PHD and Chromo Domains Directs the Rpd3S HDAC to Transcribed Chromatin}

\author{
Bing Li, ${ }^{1}$ Madelaine Gogol, ${ }^{1}$ Mike Carey, ${ }^{1,2}$ Daeyoup Lee, ${ }^{1 *}$ Chris Seidel, ${ }^{1}$ Jerry L. Workman ${ }^{1} \dagger$
}

Nucleosomes must be deacetylated behind elongating RNA polymerase II to prevent cryptic initiation of transcription within the coding region. RNA polymerase II signals for deacetylation through the methylation of histone $\mathrm{H} 3$ lysine 36 (H3K36), which provides the recruitment signal for the Rpd3S histone deacetylase complex (HDAC). The recognition of methyl H3K36 by Rpd3S requires the chromodomain of its Eaf3 subunit. Paradoxically, Eaf3 is also a subunit of the NuA4 acetyltransferase complex, yet NuA4 does not recognize methyl H3K36 nucleosomes. In Saccharomyces cerevisiae, we found that methyl H3K36 nucleosome recognition by Rpd3S also requires the plant homeobox domain (PHD) of its Rco1 subunit. Thus, the coupled chromo and PHD domains of Rpd3S specify recognition of the methyl H3K36 mark, demonstrating the first combinatorial domain requirement within a protein complex to read a specific histone code.

$\mathrm{H}$ istone modifications are important for almost all DNA-related processes, but studies of the role of histone methylation in transcription regulation have become a major focus in the field (1). Methylated lysines K4me, $\mathrm{K} 36 \mathrm{me}$, and $\mathrm{K} 79 \mathrm{me}$ are enriched around regions of active transcription $(2,3)$. These methylation marks do not simply facilitate transcription, because $\mathrm{K} 4 \mathrm{me}$ does not affect transcription per se in vitro (4). Moreover, for the majority of the yeast genome, transcription occurs normally in the absence of K4me, K36me, or K79me, whereas

\footnotetext{
${ }^{1}$ Stowers Institute for Medical Research, 1000 East 50th Street, Kansas City, MO 64110, USA. ${ }^{2}$ Department of Biological Chemistry, David Geffen School of Medicine, University of California Los Angeles, 10833 LeConte Avenue, Los Angeles, CA 90095, USA.

*Present address: Department of Biological Sciences, Korea Advanced Institute of Science and Technology, Daejeon 305-701, South Korea.

†To whom correspondence should be addressed. E-mail: jlw@stowers-institute.org
}

methylation appears to be dependent on active transcription (5). These observations imply that methylation acts in maintaining the architecture of transcribed-chromatin templates, rather than directly facilitating transcription. Consistent with this hypothesis, recent studies demonstrate that $\mathrm{K} 36 \mathrm{me}$ is recognized by the chromodomain of its Eaf3 subunit $\left(\mathrm{CHD}_{\text {Eaf3 }}\right)$ within $\mathrm{Rpd} 3 \mathrm{~S}$, thereby tethering Rpd3S to the coding region of actively transcribed genes. Once targeted, Rpd3S creates a hypoacetylated state, which in turn suppresses transcription initiated within the body of the gene (6-8).

Many chromatin-related complexes contain multiple domains that can recognize specific histone marks, but their contributions to the specificity and function of the complexes remain elusive. We discovered a critical role of the plant homeobox domain of the Rcol subunit $\left(\mathrm{PHD}_{\mathrm{Rcol}}\right)$ in Rpd3S targeting. Our data suggest that $\mathrm{CHD}_{\text {Eaf3 }}$ and $\mathrm{PHD}_{\mathrm{Rcol}}$ contribute combinatorially to the overall affinity and specificity of Rpd3S for its nu- cleosomal targets, and both domains are essential for Rpd3S-mediated control of global-acetylation levels at transcribed chromatin in vivo.

Previous studies have demonstrated that the $\mathrm{CHD}_{\text {Eaf3 }}$ in Rpd3S preferentially binds to K36me2 histone peptides $(6,7)$. We wanted to further test Rpd3S binding in a nucleosomal context. To measure the binding of $\mathrm{Rpd} 3 \mathrm{~S}$ to modified nucleosomes, we developed an assay. Reconstituted recombinant nucleosomes were immobilized on magnetic beads, sequentially modified, and then washed to remove the modifying enzymes. The resulting nucleosomes were released by restriction digestion, as illustrated in Fig. 1A. We examined the following combinations of modifications: mock-modified, acetylated by Spt-Ada-Gen5-acetyltransferase and $\mathrm{NuA} 4$, methylated at $\mathrm{H} 3 \mathrm{~K} 36$ by the recombinant hSet2 (Fig. 1, B and C), and both acetylated and methylated. We found that Rpd3S bound to methylated nucleosomes with a higher affinity than to either unmodified or acetylated nucleosomes, and acetylation further enhanced the binding of Rpd3S to the methylated nucleosomes (Fig. 1D). However, Rpd3S was unable to bind the 147-base pair nucleosome lacking linker DNA, even when the appropriate modifications were present (Fig. 1E). The recognition of K36me by Rpd3S is specific because H3K79me does not stimulate Rpd3S nucleosomal binding (fig. S3).

$\mathrm{CHD}_{\text {Eaf3 }}$ plays a pivotal role in recruiting Rpd3S to chromatin, both in vitro and in vivo (6-8). We constructed a mutant Rpd3S complex in which $\mathrm{CHD}_{\mathrm{Eaf} 3}$ was deleted (eaf3 $\Delta$ chd). The wildtype (WT) and mutant complexes were purified by tandem affinity purification (TAP)-tagged Rcol. Based on silver-stained gels, the deletion of $\mathrm{CHD}_{\text {Eaf3 }}$ did not affect the integrity of Rpd3S (Fig. 2A). This deletion did, however, substantially reduce the affinity of Rpd3S for nucleosomes (Fig. 2B), as only weak binding of the mutant 


\section{Science}

\section{Revisiting the Role of the Mother Centriole in Centriole Biogenesis}

A. Rodrigues-Martins, M. Riparbelli, G. Callaini, D. M. Glover and M. Bettencourt-Dias

Science 316 (5827), 1046-1050.

DOI: 10.1126/science.1142950originally published online April 26, 2007

ARTICLE TOOLS

SUPPLEMENTARY MATERIALS

REFERENCES

PERMISSIONS http://science.sciencemag.org/content/316/5827/1046

http://science.sciencemag.org/content/suppl/2007/04/24/1142950.DC1

This article cites 29 articles, 12 of which you can access for free http://science.sciencemag.org/content/316/5827/1046\#BIBL

http://www.sciencemag.org/help/reprints-and-permissions

Science (print ISSN 0036-8075; online ISSN 1095-9203) is published by the American Association for the Advancement of Science, 1200 New York Avenue NW, Washington, DC 20005. The title Science is a registered trademark of AAAS.

American Association for the Advancement of Science 